\title{
Subarachnoid contrast extravasation after intravenous and intra-arterial reperfusion therapy
}

Figure Postprocedure CT and MRI and 2-day follow-up CT
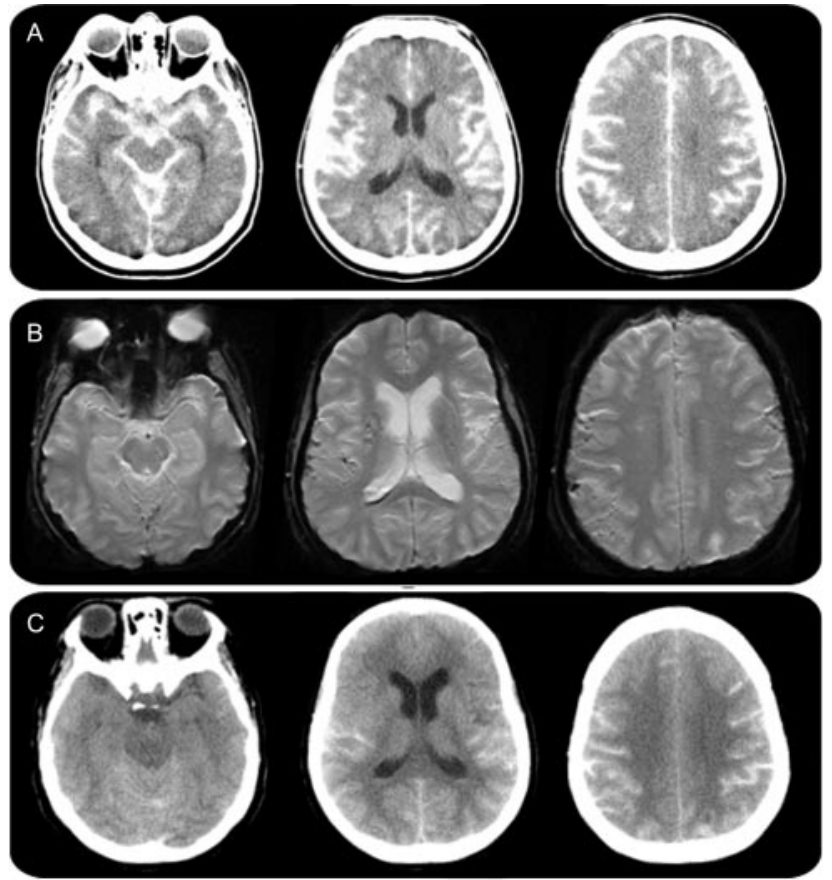

After successful recanalization, the subarachnoid space appeared hyperdense on CT (A), but gradient echo MRI (B) did not demonstrate corresponding susceptibility artifact. The CT hyperdensity cleared over 2 days (C). There was no renal impairment or postprocedural encephalopathy. CT angiography and angiogram totaled approximately 150 milliliters of contrast (Ultravist 240, Bayer).

A 63-year-old woman presented with acute hemiparesis from right middle cerebral artery occlusion. After IV tissue plasminogen activator and intra-arterial treatment with Merci retriever, balloon angioplasty, and urokinase, CT demonstrated diffuse subarachnoid hyperdensity (figure, A). Because the patient appeared clinically improved, subarachnoid contrast extravasation rather than hemorrhage was suspected. Hounsfield units may not reliably distinguish between diluted contrast and blood, but absence of susceptibility on gradient echo MRI (figure, B) and rapid contrast clearance on serial CT (figure, C) support the correct diagnosis. ${ }^{1}$ Potential etiologies include blood-brain barrier breakdown ${ }^{2}$ and microvascular injury due to device manipulation.

Wolfgang Leesch, MD, New York, NY; Brian L. Edlow, MD, Albert J. Yoo, MD, David M. Greer, MD, MA, Boston, MA

Disclosure: Dr. Leesch and Dr. Edlow report no disclosures. Dr. Yoo has received research funding from Penumbra, Inc. Dr. Greer receives royalties from the publication of Acute Ischemic Stroke: An Evidence-Based Approach (Wiley and Sons, 2007); has received research support and speaker honoraria from Boehringer Ingelheim; and has served as a consultant in medical legal cases.

Address correspondence and reprint requests to Dr. Wolfgang Leesch, Institute for Neurology and Neurosurgery, Center for Endovascular Surgery, Roosevelt Hospital, 1000 Tenth Avenue, Suite 10G, New York, NY 10019; wleesch@chpnet.org

1. Greer DM, Koroshetz WJ, Cullen S, Gonzalez RG, Lev MH. Magnetic resonance imaging improves detection of intracerebral hemorrhage over computed tomography after intra-arterial thrombolysis. Stroke 2004;35:491-495.

2. Eckel TS, Breiter SN, Monsein LH. Subarachnoid contrast enhancement after spinal angiography mimicking diffuse subarachnoid hemorrhage. AJR Am J Roentgenol 1998;170:503-505. 


\section{Neurology}

\section{Subarachnoid contrast extravasation after intravenous and intra-arterial reperfusion therapy}

Wolfgang Leesch, Brian L. Edlow, Albert J. Yoo, et al.

Neurology 2010;74;1328

DOI 10.1212/WNL.0b013e3181d9ed46

\section{This information is current as of April 19, 2010}

\section{Updated Information \& Services}

References

Subspecialty Collections

Permissions \& Licensing

\section{Reprints}

including high resolution figures, can be found at: http://n.neurology.org/content/74/16/1328.full

This article cites 2 articles, 1 of which you can access for free at: http://n.neurology.org/content/74/16/1328.full\#ref-list-1

This article, along with others on similar topics, appears in the following collection(s):

All Cerebrovascular disease/Stroke

http://n.neurology.org/cgi/collection/all_cerebrovascular_disease_strok e

\section{All Imaging}

http://n.neurology.org/cgi/collection/all_imaging

Critical care

http://n.neurology.org/cgi/collection/critical_care

Embolism

http://n.neurology.org/cgi/collection/embolism

Subarachnoid hemorrhage

http://n.neurology.org/cgi/collection/subarachnoid_hemorrhage

Information about reproducing this article in parts (figures,tables) or in its entirety can be found online at:

http://www.neurology.org/about/about_the_journal\#permissions

Information about ordering reprints can be found online:

http://n.neurology.org/subscribers/advertise

Neurology ${ }^{\circledR}$ is the official journal of the American Academy of Neurology. Published continuously since 1951, it is now a weekly with 48 issues per year. Copyright . All rights reserved. Print ISSN: 0028-3878. Online ISSN: 1526-632X.

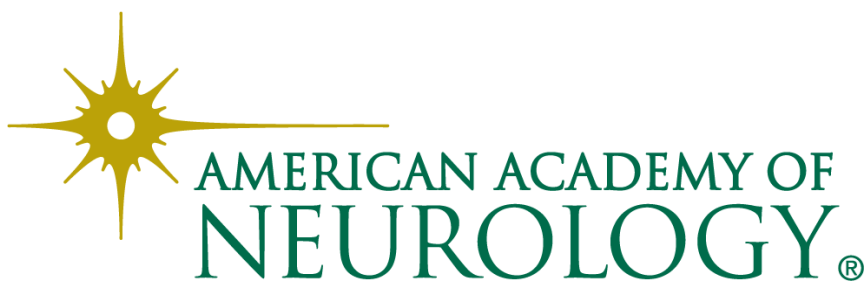

